\title{
Dynamic Scaling in Vacancy-Mediated Disordering
}

\author{
B. Schmittmann, R.K.P. Zia, and Wannapong Triampo \\ Department of Physics and Center \\ for Stochastic Processes in Science and Engineering, \\ Virginia Tech, Blacksburg, V.A. 24061-0435, USA
}

Received 1 November 1999

\begin{abstract}
We consider the disordering dynamics of an interacting binary alloy with a small admixture of vacancies which mediate atom-atom exchanges. Starting from a perfectly phase-segregated state, the system is rapidly heated to a temperature in the disordered phase. A suitable disorder parameter, namely, the number of broken bonds, is monitored as a function of time. Using Monte Carlo simulations and a coarse-grained field theory, we show that the late stages of this process exhibit dynamic scaling, characterized by a set of scaling functions and exponents. We discuss the universality of these exponents and comment on some subtleties in the early stages of the disordering process.
\end{abstract}

\section{Introduction}

The dynamics of binary alloys, undergoing mixing or segregation processes, is a venerable problem in the physics and materials science communities [1]. Much interest has focused specifically on the dynamics of phase ordering and domain growth, following a rapid quench below the coexistence curve. Starting from an initial non-equilibrium configuration, the system evolves towards its final equilibrium state, giving rise to fundamental questions in both disciplines. Crucial to metallurgy, the domain morphology is intimately linked to structural stability while statistical physics focuses on the universal, self-similar aspects of its temporal evolution, manifested in characteristic growth exponents and dynamic scaling.

A key ingredient in the study of these processes is the mechanism by which two particles exchange positions. From the viewpoint of modelling and simulation purposes, direct particle-particle exchanges obviously lead to the simplest codes and coarse-grained equations. Moreover, invoking universality, it is expected that the details of the microscopic mechanisms do not affect large-wavelength, long-time properties, such as growth exponents and universal scaling functions. In this spirit, Kawasaki dynamics and the Cahn-Hilliard equation have been extensively used to describe phase ordering in binary alloys. However, in most real solids, microscopic atom-atom exchanges can be mediated by a variety of processes [2], with direct exchanges playing a rather small role since steric hindrance tends to create large energy barriers. The most common mechanisms involve exchanges with defects, such as vacancies or in- terstitial sites. For these reasons, alloys have often been modelled by three-state models [3] whose dynamics is controlled by atom-vacancy exchanges, with direct exchanges being completely forbidden. Two problems in particular have attracted considerable attention: first, the effect of vacancies on phase separation and domain growth $[4,5]$, and second, their role in atomic interdiffusion $[6,7]$. A number of studies have also addressed vacancy-mediated ordering in a variety of antiferromagnetic alloys [8], as well as surface modes of unstable droplets in a stable vapor phase [9].

In this review, we will describe a third aspect of defect-mediated dynamics, namely, the "inverse" of the phase ordering problem. Instead of studying the growth of order in response to a sudden temperature decrease (a "quench"), we focus on the disordering of a finite system, following a rapid increase in temperature. Starting with a zero-temperature ferromagnetic configuration, i.e., a perfectly phase-segregated system with sharp interfaces, we monitor how the interfaces "roughen" and how particles of one species are transported into regions dominated by the other species. Clearly, if the final temperature is sufficiently high, the interfaces will eventually dissolve completely, leaving us with a homogeneous final state. Several questions emerge quite naturally: Are there characteristic time scales on which the disordering takes place, and how do they depend on system size, temperature and other control parameters? How do local density profiles and correlation functions evolve with time? Are there any scaling regimes, and what are the appropriate scaling variables? How do these features respond to changes in the relative concentrations of vacancies and alloy com- 
ponents?

In the following, we address some of these questions in a simple model for defect-mediated interface destruction and bulk disordering. We consider a symmetric (Ising-like) binary alloy of $\mathrm{A}$ and $\mathrm{B}$ atoms which is diluted by a very small number of vacancies (defects), reflecting the minute vacancy concentrations (of the order of $10^{-5}$ ) found in most real systems. Following an upquench from zero to a finite temperature $T$, the vacancies act as "catalysts" for the disordering process, exchanging with neighboring particles according to the usual energetics of the (dilute) Ising model. The particles themselves form a passive background whose dynamics is slaved to the defect motion. Thus, this system corresponds to a real material in which the characteristic time scale for vacancy diffusion is much faster than the ordinary bulk diffusion time. While vacancies are typically distributed uniformly in the bulk, certain defects may prefer to accumulate at the interfaces. Thus, the number of defects is not necessarily extensive in system size.

While we allow for some variation in the vacancy number, we consider equal concentrations of $\mathrm{A}$ and $\mathrm{B}$ atoms. Thus, our work forms a natural complement to the only other study [10] of vacancy-mediated disordering in the literature. There, the alloy composition is chosen highly asymmetric: $95 \%$ of A atoms versus only $5 \%$ for the $\mathrm{B}$ species, with a single vacancy. Thus, the $\mathrm{A}$ atoms form a matrix for a B-precipitate. The alloy is first equilibrated at a very low temperature, so that small clusters of B atoms are present. It is then rapidly heated to a higher temperature, and the number and size of B clusters are monitored. Three different scenarios are observed, depending on whether the final temperature is below the miscibility gap, above the miscibility gap but below $T_{c}$, or above $T_{c}$. In the first case, the precipitates remain compact. They dissolve partially at first, but then equilibrate again by coarsening. In the second case, the precipitates also remain compact but eventually dissolve completely, mostly through "evaporation" from their surfaces. In the third case, the clusters decompose rapidly ("explode") into a large number of small fragments which then disappear diffusively.

The complete or partial mixing of two materials at an interface plays a key role in many physical processes, such as corrosion or erosion phenomena [11]. We mention just two applications with huge technological potential for device fabrication. The first concerns nanowire etching by electron beam lithography [12]: If a thin film of platinum is deposited on a silicon wafer, interdiffusion of $\mathrm{Pt}$ and $\mathrm{Si}$ produces a mixing layer. If this layer is heated locally by, e.g., exposure to a conventional electron beam, silicides, such as $\mathrm{Pt}_{2} \mathrm{Si}$ and PtSi, form. The unexposed platinum can subsequently be etched away, leaving conducting nanoscale structures behind. The second example concerns mesoscopic superlattice structures, consisting of alternating magnetic and nonmagnetic metallic layers. If adjacent magnetic layers couple antiferromagnetically, the application of a large uniform magnetic field to these layered structures results in giant magnetoresistance [13]. However, the performance of these devices requires precisely engineered layer thicknesses and interfaces, and can be significantly affected by disorder [14], including interdiffusion or interfacial fluctuations.

While motivated by these applications, our study can only form a baseline here, for further work on more realistic models. However, it also has some rather fundamental implications. First, it serves as a testing ground for a basic problem in statistical physics, namely, how a system approaches its final steady state, starting from an initial non-stationary configuration. A second view of our study addresses the effect of a random walker on its background medium. Each move of a vacancy rearranges the background atoms slightly, leaving a trail behind like a child running across a sandy beach. In the simplest case, the walker is purely Brownian. In our language, this corresponds to an upquench to infinite temperature, $T=\infty$, where energy barriers are completely irrelevant. In this case, there is no feedback from the background to the local motion of the vacancy. Nevertheless, each displaced atom displays its own intriguing dynamics (mainly in $d=2$ ) $[16,17]$. To study the collective behavior of the atoms, [18] explored a lattice filled with just two species of (indistinguishable) particles. Beyond this simple case is a system heated to finite temperatures. Since the background affects the vacancy through the local energetics associated with the next move, no exact solutions are known. Instead, progress relies mainly on simulations.

The key result of our study is the observation of three distinct temporal regimes, separated by two crossover times, provided the final temperature is not too close to $T_{c}$. The intermediate and late stages of the disordering process exhibit dynamic scaling, with characteristic exponents and scaling functions that are computed analytically. For the system sizes considered here, a clear breakdown of these scaling forms is observed for temperatures within about $10 \%$ of $T_{c}$. We argue that this occurs when the correlation length becomes comparable to the system size. In contrast, the early stage of the disordering process consists of interfacial destruction in a highly anisotropic manner [17].

This article is organized as follows. We first in- 
troduce our model and define an appropriate "disorder" parameter. We then review the purely diffusive case, corresponding to $T=\infty$ [18]. Simulation results for two-dimensiononal systems and an exactly soluble mean-field theory in general dimension are discussed. In Section IV, we present a number of new results, concerning upquenches to finite temperatures where particle-particle interactions come into play. We conclude with some comments on the effect of external fields, upquenches to temperatures $T<T_{c}$, and details concerning interfacial destruction in the early stages.

\section{The Model}

In this section, we describe the dilute Ising model underlying our Monte Carlo simulations. It is defined on a two-dimensional $(d=2)$ square lattice of dimension $L \times L$, with sites denoted by a pair of integers, $\mathbf{r} \equiv(x, y)$. The boundary conditions are fully periodic in all directions. To model the two species of particles, black ("spin up") and white ("spin down", displayed as gray in the figures), and the vacancies, we introduce a spin variable $\sigma_{\mathbf{r}}$ at each site which can take three values: +1 $(-1)$ if the site is occupied by a black (white) particle, and $\sigma_{\mathbf{r}}=0$ if it is empty. Multiple occupancy is forbidden. The numbers of black $\left(N^{+}\right)$and white $\left(N^{-}\right)$ particles are conserved and differ by at most 1 . The number of vacancies, $M$, is much smaller: $M \ll N^{+}$. In fact, most of our simulations will be restricted to $M=1$, to model the minute vacancy concentrations in real systems. In our analytic work, we will also consider a more general case, where $M$ is allowed to vary with system size according to $M \propto L^{\gamma}$. Different values of the vacancy number exponent $\gamma \in[0, d]$ will be discussed. Clearly, the single vacancy case corresponds to $\gamma=0$.

The particles and vacancies interact with one another according to a dilute Ising model:

$$
\mathcal{H}\left[\sigma_{\mathbf{r}}\right]=-J \sum_{\left\langle\mathbf{r}, \mathbf{r}^{\prime}\right\rangle} \sigma_{\mathbf{r}} \sigma_{\mathbf{r}^{\prime}}
$$

with a ferromagnetic, nearest-neighbor coupling $J>0$. Since the dilution is so small, the behavior of the system is that of the ordinary (non-dilute) two-dimensional Ising model. Thus, it has a phase transition, from a disordered to a phase-segregated phase, at the Onsager critical temperature, $T_{c}=2.267 \ldots J / k_{B}$ [19]. The ground state is doubly degenerate. It consists of a strip of black and a strip of white particles, each filling half the system, separated by two planar interfaces running parallel to a lattice axis. Since the particle-particle interactions are ferromagnetic, the few vacancies accumulate at the interfaces.
Next, we turn to the dynamics of the model. Only particle-hole exchanges are allowed and performed with the usual Metropolis [20] rate: $\min \{1, \exp (-\beta \Delta \mathcal{H})\}$, where $\Delta \mathcal{H}$ is the energy difference of the system before and after the jump, and $\beta=1 /\left(k_{B} T\right)$ is the inverse temperature. The initial configuration of the system is perfectly phase-segregated, with two interfaces chosen to lie along the $x$-axis. The vacancies are located at random positions along one of the interfaces. Since this is technically a zero-temperature configuration, while the dynamics occurs at temperature $T>0$, the vacancies will move around, disordering the interfaces and, if $T>T_{c}$, dissolving them eventually. The final steady state is an equilibrium state of the usual two-dimensional Ising model. Therefore, many of its properties are exactly known [21].

In our simulations, the system sizes range from $L=30$ to 60 . The final temperature $T$, measured in units of the Onsager temperature $T_{c}$, varied between $1.1 T_{c}$ and infinity. Our data are averages over $10^{2}$ to $10^{4}$ realizations (or runs) depending on the desired quality of the data. The time unit is one Monte Carlo step (MCS) which corresponds to $M$ attempted particle-hole exchanges. All systems investigated equilibrate after about $10^{8}$ MCS.

To monitor the evolution of the system, we measure a "disorder parameter", defined as the average number of black and white nearest-neighbor pairs, $\mathcal{A}(L, t)$, as a function of (Monte Carlo) time $t$. This quantity is easily related to the Ising energy,

$$
\mathcal{A}(L, t)=\frac{d}{2} L^{d}+\frac{1}{2 J}\langle\mathcal{H}\rangle+O\left(L^{\gamma}\right)
$$

where $\langle\cdot\rangle$ denotes the configurational average over runs. The correction is due to the vacancies and remains much smaller than the two leading terms. More detailed information is carried by the local hole and magnetization densities, defined respectively as

$$
\begin{aligned}
\phi(\mathbf{r}, t) & \equiv\left\langle\delta_{\sigma_{\mathbf{r}}, 0}\right\rangle \\
\psi(\mathbf{r}, t) & \equiv\left\langle\sigma_{\mathbf{r}}\right\rangle
\end{aligned}
$$

The Kronecker- $\delta$ ensures that lattice site $\mathbf{r}$ is occupied by the vacancy. Non-zero values of $\psi(\mathbf{r}, t)$ indicate an excess of white or black particles at lattice site $\mathbf{r}$, which is obviously a sensitive measure of the disordering process. The full time dependence of these densities can in general only be computed within a mean-field approach. However, their stationary forms are easily found from exactly known properties of the two-dimensional Ising model. 


\section{Brownian Vacancies: $T=\infty$}

In this Section, we focus on the simplest case, namely, upquenches to infinite temperature. As a consequence, the nearest-neighbor coupling $J$ plays no role at all, and all attempted particle-hole exchanges are executed with unit rate. Thus, the vacancies perform a Brownian random walk, regardless of their local environment. We first summarize our simulation results which suggest the key to the mean-field analysis, namely, a separation of time scales. We then introduce a suitable set of exponents and scaling forms and show that the late stages of the disordering process display dynamic scaling. Finally, we turn to a mean-field theory and compute these exponents and scaling functions analytically.

\section{III.1 Simulation Results}

To illustrate the gradual destruction of the interfaces and the disordering of the bulk, Fig. 1 shows the evolution of a typical configuration, in a $60 \times 60$ system with a single vacancy (which is represented by a white square in the figures). At $t=0$, the vacancy is located at the interface in the center which begins to break up slowly. Eventually, the second interface also becomes affected, as more and more particles are transported into regions of opposite color, until the system finally disorders completely. For later reference, we note that the last two configurations, at $10^{7}$ and $10^{8} \mathrm{MCS}$, are both already fully random. The disordering process is clearly reflected in the number of broken bonds: $\mathcal{A}(60, t)$, shown in Fig. 2, increases from its minimum of $O(L)$ for the initial configuration at $t=0$, to $O\left(L^{2}\right)$ for the fully equilibrated system at $t=10^{7}$. One clearly distinguishes three regimes, shown schematically in the inset: an early regime (I), the intermediate, or scaling, regime (II), and finally a late or saturation regime (III) in which the system has effectively reached the steady state. Tracking the motion of the vacancy, the physical origin of these three regimes is easily identified. For early times (regime I), the vacancy is still localized in the vicinity of its starting point, far from the boundaries of the system. After a time of $O\left(L^{2}\right)$, however, the vacancy has explored the whole system and is effectively equilibrated. This marks the onset of the intermediate regime. The particle distribution is still strongly inhomogeneous here and does not equilibrate until the system enters the saturation regime. We emphasize that the second and third regimes emerge only in a finite system. In an infinite system, regime I persists for all times. Some aspects will be briefly summarized in the concluding remarks.

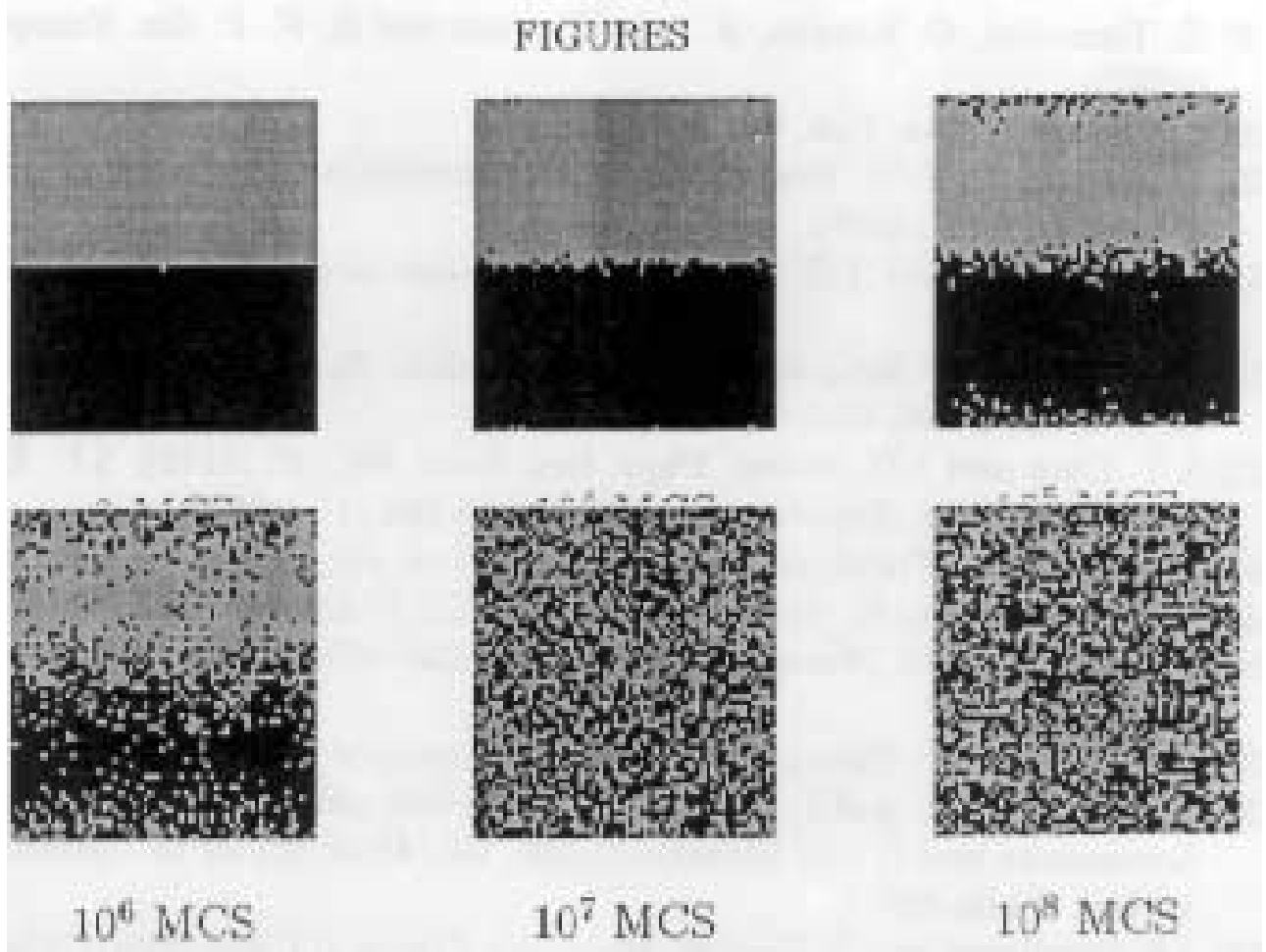

Figure 1. Sequence of snapshots showing the disordering process of a $60 \times 60$ system with $T=\infty$. The black and gray squares represent the two types of $\operatorname{particles}(\sigma= \pm 1)$ and the white square denotes the vacancy $(\sigma=0)$. The configurations were recorded after $0,10^{4}, 10^{5}, 10^{6}, 10^{7}$ and $10^{8}$ MCS. 


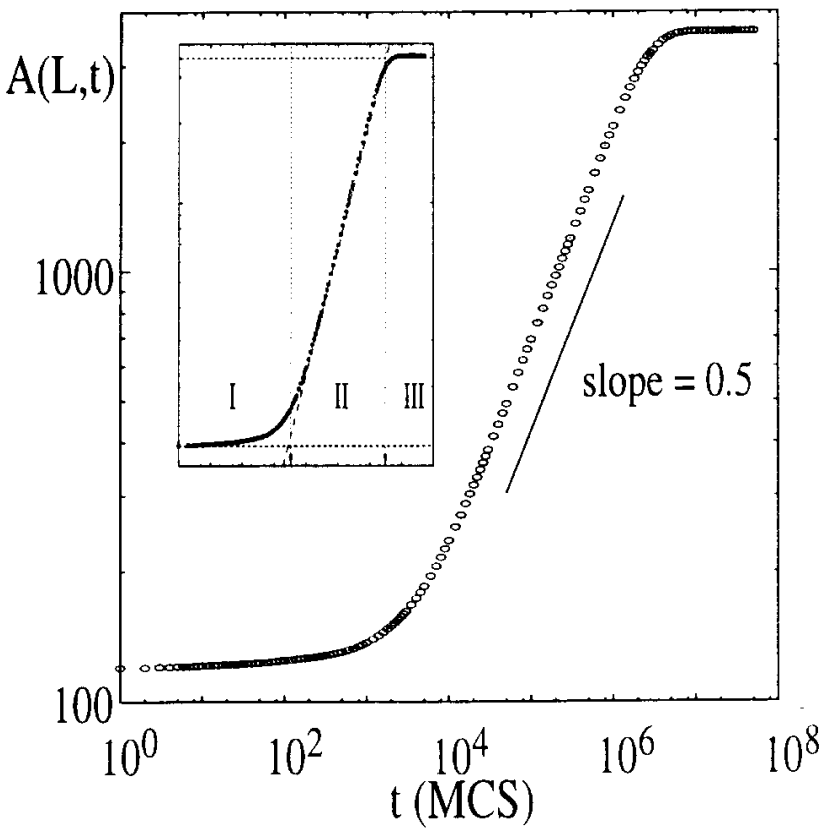

Figure 2. Plot of the total number of broken bonds, $A(L, t)$ vs $t$, for $L=60$ and $T=\infty$. It shows the emergence of an early regime (I), an intermediate, or scaling, regime (II), and a late or saturation regime (III). The straight reference line has slope 0.5 .

Our key observation is that, independent of dimension, Regimes II and III exhibit dynamic scaling. To characterize this behavior, we introduce a set of exponents: First, the saturation value of $\mathcal{A}$ scales with system size according to $\lim _{t \rightarrow \infty} \mathcal{A}(L, t) \equiv \mathcal{A}_{\text {sat }}(L) \sim L^{\alpha}$. Second, in the intermediate regime, $\mathcal{A}(L, t)$ grows as $\mathcal{A}(L, t) \sim L^{\sigma} t^{\beta}$. Finally, the two crossover times ("early" and "late") scale as $t_{e} \sim L^{z_{e}}$, and $t_{l} \sim L^{z_{l}}$. Thus, the intermediate-to-late time crossover can be summarized by the scaling form

$$
\mathcal{A}(L, t) \sim L^{\alpha} f\left(t / t_{l}\right)
$$

with a scaling function $f$ which satisfies $f(x) \simeq$ const for $x \gg 1$, and $f(x) \sim x^{\beta}$ for $x \ll 1$ (but large enough to fall within Regime II). The consistency condition $\mathcal{A}\left(L, t_{l}\right) \simeq \mathcal{A}_{\text {sat }}(L)$ yields the scaling law

$$
\sigma+z_{l} \beta=\alpha .
$$

Due to the presence of the novel exponent $\sigma$, (5) differs from the familiar $z \beta=\alpha$ which controls surface growth in, e.g., the Edwards-Wilkinson [23] or KPZ [24] models.

We first consider a single vacancy, in $d=2$. For this case, the data give $\alpha=2 \pm 0.1, \beta=0.5 \pm 0.06$, $z_{e}=2 \pm 0.2, z_{l}=4 \pm 0.2$, and $\sigma=0 \pm 0.1$. Clearly, these exponents satisfy the scaling law (5). Excellent data collapse is obtained by plotting $\mathcal{A}(L, t) / L^{\alpha}$ versus $t / L^{z_{l}}$, shown in Fig. 3 for a range of system sizes. The same indices are observed for several vacancies, provided their number remains constant as the system size $L$ is varied, corresponding to a vacancy exponent $\gamma=0$. We note briefly that the exponent $\sigma$ is required here to accomplish good data collapse in dimensions greater than 2 .

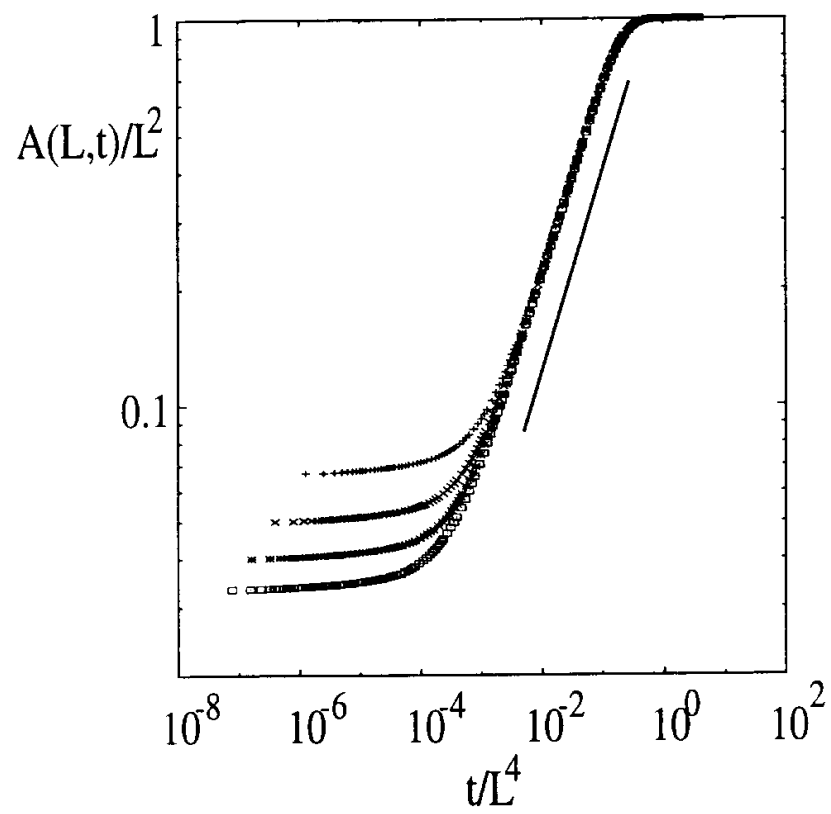

Figure 3. The scaling plot of $A(L, t) / L^{2}$ vs $t / L^{4}$ for $L=$ $30-60$ with $T=\infty$. The straight reference line has slope 0.5 .

Another natural case is $\gamma=d-1$, corresponding to a situation where the defects are initially "frozen" at the interfaces, and their number scales with the interfacial area. Such a scenario could occur in surface catalysis or in welding. Since our data are restricted to $d=2$, we examine the case $\gamma=1$, i.e., $M \propto L$. Here, the data collapse only if (i) curves corresponding to different system sizes are shifted by an $M$-dependent factor along the $\ln t$-axis, and (ii) the late crossover exponent takes on the new value $z_{l}=3 \pm 0.2$, while $\alpha$ and $\beta$ remain unchanged. This behavior suggests that the exponent $\sigma$ depends non-trivially on $\gamma$. For $\gamma=1$, the data are consistent with $\sigma=1 / 2$, so that the scaling form (4) still holds and the scaling law (5) is satisfied with the modified exponent $z_{l}$.

This concludes the discussion of our simulation results. We now turn to an analytic description of the disordering process.

\section{III.2 Mean-Field Theory}

As a first step, we summarize the exactly known results for the initial configuration and the final, fully disordered state. The analysis is easily performed in general dimension, for a hypercube of side length $L$ with 
fully periodic boundary conditions. The initial value of the disorder parameter is given by the number of broken bonds across the two initial, flat interfaces between black and white particles: $\mathcal{A}(L, t=0)=2 L^{d-1}+O(M)$. The saturation value, $\mathcal{A}_{\text {sat }}(L)=\frac{d}{2} L^{2 d} /\left(L^{d}-1\right)$ follows easily from the fact that the final steady state is completely random for the Brownian vacancy case with $T=\infty$. Thus, we can read off the exact result $\alpha=d$.

To proceed further, we derive a set of equations of motion, for the coarse-grained local hole and particle densities. Starting from the microscopic master equation, we can easily obtain evolution equations for the discrete densities, $\phi(\mathbf{r}, t)$ and $\psi(\mathbf{r}, t)$. Since there is no feedback from the particle background, the equation for $\phi(\mathbf{r}, t)$ is completely independent of $\psi(\mathbf{r}, t)$. In contrast, the motion of the particles is slaved to that of the vacancy, so that the equation for $\psi(\mathbf{r}, t)$ is inherently nonlinear and contains two-point functions of the form $\left\langle\sigma_{\mathbf{r}^{\prime}} \delta_{\sigma_{\mathbf{r}}, 0}\right\rangle$. Here, a mean-field assumption is required, to truncate these averages. Finally, we take the continuum limit, by letting the lattice constant vanish at fixed system size $L$. For simplicity, we use the same notation for discrete densities and their continuous counterparts. The position vector $\mathbf{r} \equiv\left(x_{1}, \ldots ., x_{d}\right)$ denotes a point in the hypercube $-L / 2 \leq x_{i} \leq+L / 2, i=1,2, \ldots, d$, with volume $V \equiv L^{d}$. We also retain the symbol $t$ for time, since Monte Carlo time and its coarse-grained counterpart differ only by a scale factor. After some suitable rescalings, we obtain the desired mean-field equations:

$$
\begin{aligned}
& \partial_{t} \phi(\mathbf{r}, t)=\nabla^{2} \phi(\mathbf{r}, t) \\
& \partial_{t} \psi(\mathbf{r}, t)=\phi(\mathbf{r}, t) \nabla^{2} \psi(\mathbf{r}, t)-\psi(\mathbf{r}, t) \nabla^{2} \phi(\mathbf{r}, t) .(6)
\end{aligned}
$$

The simple diffusion equation for $\phi$ reflects the Brownian random walk of the vacancies. The magnetization density $\psi$ obeys a balance equation: the first term reflects a gain, provided a vacancy is initially present and a particle "diffuses" in from a neighboring site. The second term accounts for a loss, due to a particle jumping to a vacant nearest-neighbor site. Similar equations have been discussed in the context of biased diffusion of two species [25] and vacancy-mediated interdiffusion [7]. Note that both equations take the form of continuity equations, due to the conservation laws on the particle numbers. The two densities are normalized, according to $\int_{V} \phi(\mathbf{r}, t)=M$, and $\int_{V} \psi(\mathbf{r}, t)=0$. Given fully periodic boundary conditions, these equations have to be supplemented with appropriate initial conditions. For our simulations, we choose $\phi(\mathbf{r}, 0)=\frac{M}{L^{d-1}} \delta(y)$ where $y \equiv x_{d}$, and $\psi(\mathbf{r}, 0)=2 \theta(y)-1$. The final state is, of course, trivial: $\phi(\mathbf{r}, \infty) \equiv \phi_{o}=M / V$, and $\psi(\mathbf{r}, \infty)=0$.

To solve for the hole and magnetization densities, we seek the separation of time scales, first observed in the
Monte Carlo data, in the equations (6). The hole density, with a diffusion coefficient of $O(1)$, relaxes rapidly to the final value $M / V$. By contrast, the magnetization density is essentially slaved to $\phi(\mathbf{r}, t)$ and relaxes with a diffusion "coefficient" of $O(1 / V)$. The hole density spreads diffusively and reaches the boundaries of the system after a time of $O\left(L^{2}\right)$, marking the end of the early regime. Thus, we identify the early crossover time $t_{e} \propto L^{2}$ and read off $z_{e}=2$. For later times, the vacancies are uniformly distributed over the system, so that $\phi$ may be replaced by its stationary value, $\phi_{o}=\frac{M}{V}$. Inserting this into the equation for $\psi$ results in a simple diffusion equation

$$
\partial_{t} \psi=\phi_{o} \nabla^{2} \psi
$$

with a diffusion coefficient $M / V$. Its solution, subject to the initial and fully periodic boundary conditions, is easily found:

$$
\psi(\mathbf{r}, t)=\frac{4}{\pi} \sum_{n=1}^{\infty} \frac{\sin [2 \pi(2 n-1) y / L]}{2 n-1} e^{-\epsilon t(2 n-1)^{2}}
$$

where $\epsilon \equiv 4 \pi^{2} \phi_{o} / L^{2}$. Of course, $\psi(\mathbf{r}, t)$ depends on $y$ only, reflecting the homogeneity of initial and boundary conditions in the $d-1$ transverse directions. We also note that, while this form is exact, it converges rapidly only for late times, $\epsilon t \gg 1$, corresponding to the saturation regime. There, the profile is harmonic with a rapidly decaying amplitude: $\psi(\mathbf{r}, t) \simeq$ $\frac{4}{\pi} \sin [2 \pi y / L] e^{-\epsilon t}$. Below, we will present an equivalent form with good convergence in the opposite limit.

To describe the simulation results, we need an expression for the disorder parameter $\mathcal{A}$. Recalling its connection to the average Ising energy, Eqn (2), we invoke the continuum limit of the latter $[26],\langle\mathcal{H}\rangle=$ $-d J \int_{V} \psi(\mathbf{r}, t)^{2}$, which is correct up to surface terms of $O\left(1 / L^{d-1}\right)$. Thus, we obtain

$$
\mathcal{A}(L, t)=\frac{d}{2}\left[V-\int_{V} \psi(\mathbf{r}, t)^{2} d^{d} \mathbf{r}\right] .
$$

We note that this form is manifestly extensive. Moreover, since surface terms have been dropped, the initial value of $\mathcal{A}$ is now simply 0 .

The time evolution of this quantity follows from (8):

$$
\mathcal{A}(L, t)=\frac{d}{2} V[1-S(2 \epsilon t)]
$$

where $S(\zeta) \equiv \frac{8}{\pi^{2}} \sum_{1}^{\infty} e^{-\zeta(2 n-1)^{2}} /(2 n-1)^{2}$. Since $S(0)=1$ and $S(\infty)=0$, we verify that $\mathcal{A}$ does take on the correct initial and final values. It is also consistent with our postulated scaling form, Eqn (4), provided we identify the scaling exponent $\alpha$ with $d$ 
and the scaling variable $t / t_{l}$ with $\epsilon t$. This suggests to define the late crossover time as $t_{l} \equiv 1 / \epsilon$. Collecting the dependence on system size, we identify $t_{l} \propto L^{2} / \phi_{o}=L^{d+2-\gamma}$. Hence, we read off $z_{l}=$ $d+2-\gamma$ for the scaling exponent which controls the late crossover time. Thus, $\epsilon t \gg 1$ marks the saturation regime where Eqn (4) converges well, exhibiting a simple exponential approach to the saturation value: $\mathcal{A}(L, t)=\frac{d}{2} V\left[1-\frac{8}{\pi^{2}} e^{-2 \epsilon t}+O\left(e^{-4 \epsilon t}\right)\right]$.
In contrast, the intermediate regime (II) corresponds to $\epsilon t \ll 1$. To capture the time dependence here, we re-express the infinite sum via a Poisson resummation [27]. The key advantage of this procedure is to convert a sum with rapid convergence in one limit $(\epsilon t \gg 1)$ into an equivalent sum with good convergence in the opposite $(\epsilon t \ll 1)$ limit. Deferring technical details to an Appendix, we introduce $u_{m} \equiv \pi m / \sqrt{8 \epsilon t}$ and arrive at

$$
\mathcal{A}(L, t) \simeq \frac{2 d}{\pi^{3 / 2}} V \sqrt{2 \epsilon t}\left\{1+2 \sum_{m=1}^{\infty}(-1)^{m}\left[e^{-u_{m}^{2}}-u_{m} \Gamma\left(\frac{1}{2}, u_{m}^{2}\right)\right]\right\}
$$

where $\Gamma(\bullet, \bullet)$ denotes the incomplete Gamma function. In this form, the sum over $m$ is suppressed for small $\epsilon t$. Thus, $\mathcal{A}(L, t) \propto V \sqrt{2 \epsilon t} \propto L^{d} \sqrt{M t / L^{2+d}}$, yielding the remaining indices, namely $\beta=\frac{1}{2}$ independent of dimension, and $\sigma=\frac{1}{2}(d+\gamma-2)$. It is straightforward to check that our exponents satisfy the scaling relation (5). In two dimensions, our theory predicts $z_{l}=4$ and $\sigma=0$ for $\gamma=0$, while $z_{l}=3$ and $\sigma=\frac{1}{2}$ for $\gamma=1$, in complete agreement with the Monte Carlo data.

Since the full magnetization profile contains more detailed information than the disorder parameter, one might also be interested in its form for early times, $\epsilon t \ll 1$. A similar Poisson resummation, of Eqn (8), results in

$$
\psi(\mathbf{r}, t)=\operatorname{erf}\left(\frac{\tilde{y}}{2 \sqrt{\epsilon t}}\right)+\sum_{m=1}^{\infty}(-1)^{m}\left[\operatorname{erf}\left(\frac{\tilde{y}+\pi m}{2 \sqrt{\epsilon t}}\right)+\operatorname{erf}\left(\frac{\tilde{y}-\pi m}{2 \sqrt{\epsilon t}}\right)\right]
$$

where $\tilde{y} \equiv 2 \pi y / L$. For $\epsilon t \ll 1$, this form still reflects the sharpness of the initial $\Theta$-function which is only gradually "washed out".

This concludes our discussion of the mean-field theory, for the Brownian vacancy case. We note briefly that our results remain unchanged (up to a trivial amplitude) if periodic boundary conditions are replaced by reflecting ones. We should also reiterate that our focus here rests on the intermediate and late regimes. A full microscopic treatment of the early regime is beyond the scope of this paper. For the benefit of the reader, however, a short summary is provided in the conclusions. Instead, we turn to the role of interactions.

\section{The effect of interactions:

$$
T_{c}<T<\infty
$$

When the final temperature of the system is finite, $T<\infty$, the full Hamiltonian, Eqn (1), comes into play when a vacancy attempts to move. In particular, the defects no longer perform a simple random walk, since the jump rates now carry information about the local environment, i.e., the distribution of black and white particles around the originating and receiving site. Thus, a feedback loop between vacancy and background is established, in stark contrast to the case $T=\infty$. One might expect that this has significant consequences for profiles and disorder parameter, and that dynamic scaling exponents might be modified. It is immediately obvious that we should distinguish final temperatures above criticality from those below. In the former case, the final steady-state configurations are still homogeneous, even if correlations become more noticeable. In the latter case, however, the equilibrium state itself is phase-separated, so that the only effect of the vacancies is to "soften" the initial interfaces. This aspect of vacancy-induced disordering should be particulary interesting in two dimensions, where Ising interfaces are known to be rough [28]. There, the vacancies must mediate both the development of an "intrinsic interfacial width" and the emergence of large-scale wanderings of the interface [29]. Certainly, we expect these two phenomena to take place on drastically different time scales, since they are associated with local 
vs. global disorder.

In this Section, we focus on final temperatures above criticality: $T_{c}<T<\infty$, and a single vacancy, $M=1$. Starting with a set of typical evolution pictures, we investigate the dynamic scaling properties of the disorder parameter. Since the equations of motion now become highly nonlinear, we have not been able to solve them exactly. Thus, we offer only a few comments in conclusion.

Fig. 4 shows the evolution of a typical configuration, in a $60 \times 60$ system with a single vacancy at $T=1.5 T_{c}$. Note that the MC times are the same as in Fig. 1. Again, we observe the gradual, yet eventually complete, destruction of the interfaces. In comparison with Fig. 1, however, two obvious differences emerge:
First, the system takes longer to reach the final stationary state. Second, the final configuration shows clear evidence of a finite correlation length. Both features are induced by the interactions. In particular, the disordering process is slowed down since the breaking of bonds is energetically costly. At a more quantitative level, this is documented by Fig. 5 which shows the disorder parameter, for a $60 \times 60$ system and several temperatures. One observes that the late crossover time shifts to later times as the temperature decreases. In contrast, the early crossover time appears to be less affected. Also, the saturation value of the disorder parameter decreases. However, being essentially the Ising energy, it remains extensive, with a $T$-dependent amplitude.
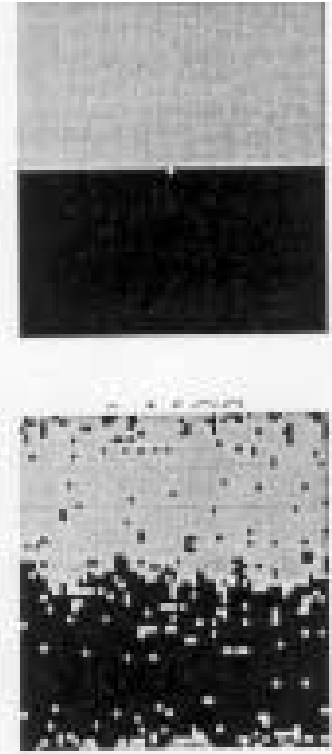

$10^{6}$ MCS
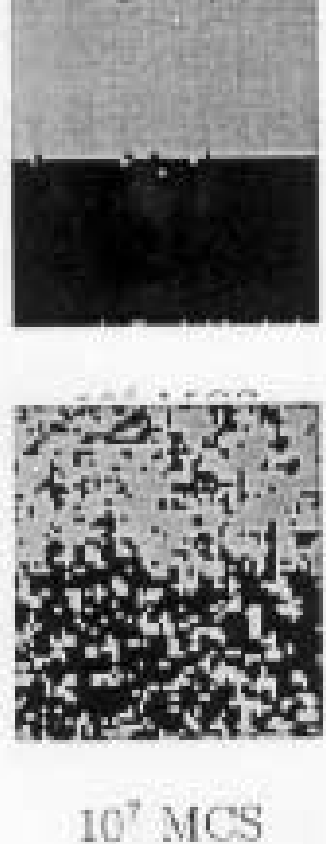
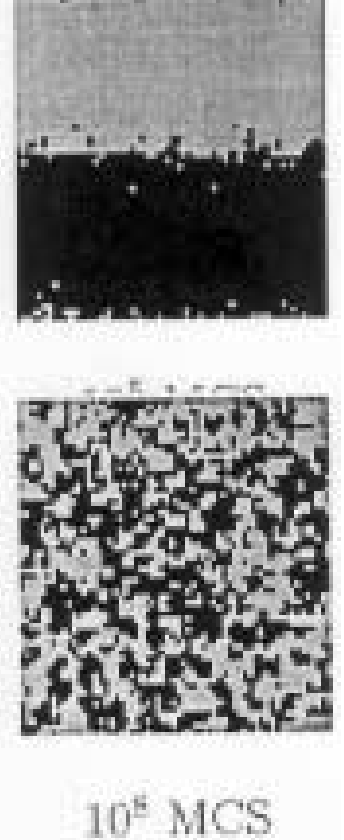

Figure 4. Sequence of snapshots showing the disordering process of a $60 \times 60$ system with $T=\infty$. The black and gray squares represent the two types of $\operatorname{particles}(\sigma= \pm 1)$ and the white square denotes the vacancy $(\sigma=0)$. The configurations were recorded after $0,10^{4}, 10^{5}, 10^{6}, 10^{7}$ and $10^{8}$ MCS.

It is natural to ask whether the scaling forms found in the previous section still hold. In Fig. 6, we present the scaling plots for the disorder parameter, i.e., we show $\mathcal{A}(L, t) / L^{d}$ plotted vs. $t / L^{4}$. Each graph corresponds to a different temperature: $T=3.5 T_{c}$ (Fig. 6a), $T=1.5 T_{c}$ (Fig. 6b), and $T=1.1 T_{c}$ (Fig. 6c). Focusing on Fig. 6a, it is quite evident that the data collapse just as well for $T=3.5 T_{c}$ as for $T=\infty$ (cf. Fig. 3). The three temporal regimes are well separated and easy to distinguish. In the intermediate regime, the disorder parameter follows the $t^{1 / 2}$ power law, over more than three decades. Thus, the data at this temperature still obey the scaling form (4), with the same set of exponents. However, for the next temperature, $T=1.5 T_{c}$
(Fig. 6b), the range of perfect data collapse begins to shrink: the curve for the smallest system size $(L=30)$ merges noticeably later than in Fig. 6a. Also, the power law no longer persists over such a wide region, and the exponent $\beta$ becomes more difficult to determine. Still, the larger system sizes collapse rather well, so that there is still a well-defined scaling function even if its form appears to change. Finally, Fig. 6c shows the data for $T=1.1 T_{c}$ : here, the imposed scaling form manifestly fails to match the data. 


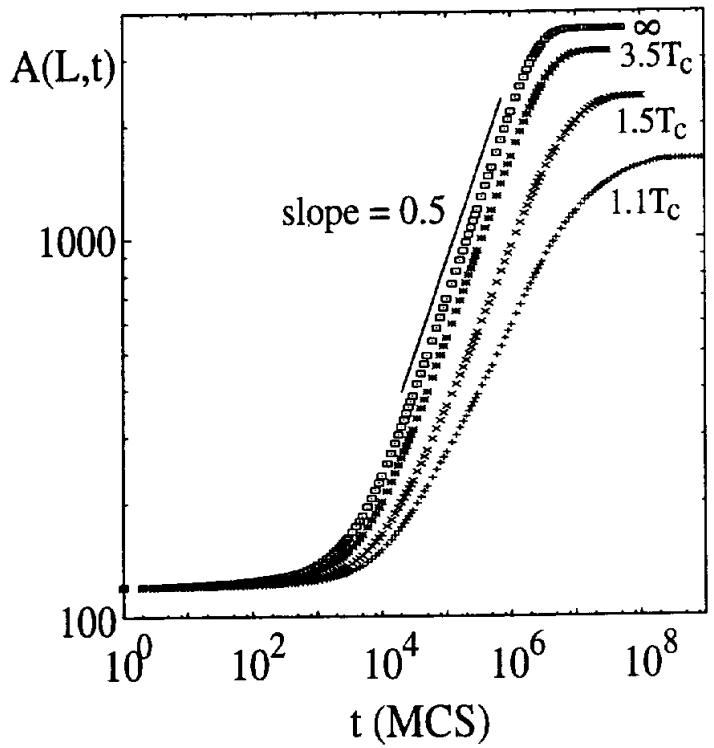

Figure 5. $A(L, t)$ vs $t$ for $L=60$ with $T=\infty, 3.5 T_{c}, 1.5 T_{c}$ and $1.1 T_{c}$.
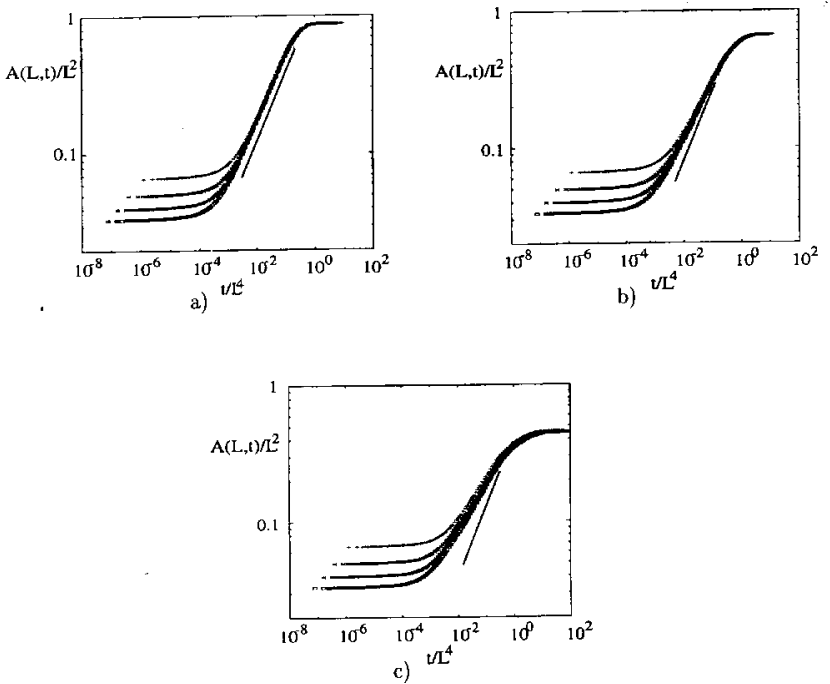

Figure 6. The scaling plot of $A(L, t) / L^{2}$ vs $t / L^{4}$ for $L=30-60$ with (a) $T=3.5 T_{c}$, (b) $T=1.5 T_{c}$ and (c) $T=1.1 T_{c}$. The straight reference lines have slope 0.5 .

We conclude that, for temperatures not too close to the critical temperature $T_{c}$, the dynamic scaling form of the purely diffusive case, Eqn (4), still holds. In two dimensions, we identify the saturation exponent $\alpha=2$ and, for a single vacancy, the late crossover exponent $z_{l}=4$. The scaling function itself, however, develops more curvature in the intermediate regime, so that it becomes more difficult to extract a pure power law, $t^{\beta}$. One might be tempted to fit the data to a pure power law, resulting in a temperature-dependent exponent $\beta$, noticeably smaller than 0.5 . However, we believe that there is little physical support for such a drastic change in scaling properties, especially in the disordered phase of the Ising model. Instead, we conjecture that $\beta=1 / 2$ is still valid but that $T$-dependent corrections-to-scaling begin to play a stronger role. In support, we checked that the data for $T=1.5 T_{c}$, in the earlier part of regime II, can be well fitted by a power law with an exponential correction, i.e., $\mathcal{A}(L, t) / L^{2} \propto t^{1 / 2}\left\{1+c_{1}(T) \exp \left[-c_{2}(T) t / L^{4}\right]\right\}$. This form is gleaned from Eqn (11), by keeping the leading and first sub-leading term but allowing for two temperature-dependent fit parameters $c_{1}(T)$ and $c_{2}(T)$. Of course, we should anticipate significant changes in the scaling behavior upon entering the critical region. Since $1.1 T_{c}$ is "at the doorstep" of the latter, some precursors such as an increased correlation length begin to make their influence felt.

In the following, we comment briefly on some analytic results. First, we can easily check that the saturation values, $\mathcal{A}_{\text {sat }}(L)$, are consistent with exact results for the two-dimensional Ising value. Table 1 shows this comparison for a $60 \times 60$ system with a single vacancy, at several temperatures. $\mathcal{A}_{\text {sat }}^{M C}$ is the measured saturation value of the disorder parameter. $\mathcal{A}_{\text {sat }}^{T H}$ is calculated on the basis of Eqn (2), where $\langle\mathcal{H}\rangle$ is the exact bulk value for the average energy of the usual Ising model [21]. The effect of the vacancy has been neglected. The agreement is very good, within the statistical errors of our data. The discrepancies are of course largest for the last column, with $T$ closest to $T_{c}$.

Finally, since $\langle\mathcal{H}\rangle$, and therefore $\mathcal{A}_{\text {sat }}(L)$, are extensive, we still have an exact value for the saturation exponent $\alpha=d$.

The time-dependence of the disorder parameter poses a much more difficult problem. It is of course possible to obtain the temperature-dependent generalization of Eqns (6), by averaging the microscopic master equation, followed by a mean-field approximation and a naive continuum limit. For a dynamics slightly different from ours, this procedure was followed in [5]. However, these equations are no longer easily soluble: First, both of them are inherently nonlinear, due to interactions. Second, the equation for the local hole density, $\phi(\mathbf{r}, t)$, no longer decouples from the magnetization $\psi(\mathbf{r}, t)$, since the background feeds back into the motion of the vacancy. Thus, it is not apparent how a clean time scale separation could emerge from these equations. As a consequence, we are reduced to numerical solutions or approximate techniques, such as a high temperature expansion. Preliminary results, based on the latter, provide some support for our conjecture that $\beta=1 / 2$ is still valid and that larger corrections-toscaling are responsible for the curvature in the scaling function [30].

To summarize this section briefly, our data indicate that, for temperatures not too close to the critical temperature $T_{c}$, the dynamic scaling form of the purely diffusive case still holds, even though corrections-toscaling become more noticeable. In contrast, for $T$ near $T_{c}$ we observe a clear breakdown of these scaling forms. 
Table 1. Comparison of exact and measured values of the saturated disorder parameter for several temperatures.

\begin{tabular}{||l|c|c|c|c||}
\hline$T / T_{c}$ & $\infty$ & 3.5 & 1.5 & 1.1 \\
\hline $\mathcal{A}_{\text {sat }}^{T H}$ & 3600.0 & 3134.6 & 2367.0 & 1602.9 \\
\hline $\mathcal{A}_{\text {sat }}^{M C}$ & 3598.6 & 3134.0 & 2368.9 & 1615.7 \\
\hline
\end{tabular}

\section{Concluding Remarks}

To summarize, we have analyzed the vacancy-driven disordering process of an initially phase segregated binary system, which is rapidly heated to a temperature $T$ above criticality. The number of vacancies scales with system size as $L^{\gamma}$, where the vacancy exponent $\gamma$ is a parameter. To quantify the evolution of the system, we measure the number of broken bonds, $\mathcal{A}(L, t)$, as a function of time. This "disorder parameter" allows us to identify three temporal regimes, distinguished by the distribution of the vacancies through the system. Our key result is that the late stages of this process exhibit dynamic scaling. A set of exponents $\left\{z_{e}, z_{l}, \alpha, \sigma ; \beta\right\}$ can be defined, characterizing, respectively, the system size dependence of two crossover times, the final saturation value of the disorder parameter, and its amplitude in the intermediate regime. The temporal growth of $\mathcal{A}$ during the latter regime is captured by the exponent $\beta$. If the final temperature is infinite, i.e., $T=\infty$, the motion of the vacancies is a simple Brownian random walk, and all indices can be computed analytically, in excellent agreement with the data. In particular, we find that the typical time scale $t_{l} \sim L^{z_{l}}$, which controls the crossover between increasing disorder and saturation, is set by $z_{l}=2+d-\gamma$, and thus depends explicitly on both the space and vacancy dimensionalities, $d$ and $\gamma$. Measurements of $z_{l}$ can therefore provide information about the vacancy distribution in a sample. In the most familiar case, standard vacancy diffusion in solids, the number of vacancies is extensive $(\gamma=d)$, so that the well-known result $z_{l}=2$ is reproduced [2]. In contrast, in two dimensions we find $z_{l}=4$ for a single vacancy $(\gamma=0)$ and $z_{l}=3$ if the defects are generated at the initial interface. For finite temperature $T \gtrsim 1.5 T_{c}$, we observe that these scaling forms still hold. Even though interparticle interactions now play a role, correlations in the system are still shortranged, so that the vacancy still performs a random walk if viewed on a length scale which exceeds the correlation length, $\xi(T)$. As $T$ approaches $T_{c}$, however, $\xi(T)$ reaches $O(L)$ so that the simple random walk scenario must break down. In our simulations, significant deviations from the high-temperature scaling form appear already for $T=1.1 T_{c}$, where $\xi(T) \simeq 6$, in units of the lattice spacing [21].

All the investigations above are focused on upquenches to the disordered state. A natural extension of this project is to set the final temperature below criticality. Needless to say, new questions and interesting phenomena can be expected. In particular, the final equilibrium state is still phase separated, so that all the interfacial properties come into play. With a single vacancy in a finite system, there may be a further separation of time scales. We expect that, soon after the early stage, the "intrinsic" density profile of the interface can be built. At this point, the interfacial width, $w$, is most likely controlled by $\xi$. On the other hand, at sufficiently long times, the capillary waves will surely make their presence felt. In the $d=2$ case, the interface is always rough, so that we can always expect the latter crossover to occur. How, and if, $w(\xi, L ; t)$ scales will be of great interest. Besides dynamics and timescales, we could study the equilibrium probability profile of the vacancy, i.e., where the vacancy spends most of its time. In case more than one vacancy is present, this profile should map into a density profile for the vacancies. In either case, we expect the vacancies to be trapped, to a greater or lesser extent, at the interface. Now, if more and more vacancies are added to the system, the interface profile should be altered, since a preponderance of vacancies may significantly modify the interfacial energy. For $d=3$ Ising models, there are further interesting phenomena, associated with roughening transitions. Given that interfacial energies should depend on vacancy concentrations, how the locations (if not the nature) of such transitions are affected is a natural question. Finally, it would be extremely interesting to study the dynamic content of these systems, such as scaling properties when the upquench is set at a roughening temperature.

We conclude with a few remarks on related problems. It is obviously interesting to test the range of universality of the high-temperature scaling forms, with respect to changes in the boundary conditions and the microscopic dynamics. Returning to $T=\infty$, we have investigated several cases. First, replacing periodic by reflecting boundary conditions changes only trivial amplitudes. A more significant modification is the addition of a bias $E$, such as a gravitational or electric field [31]: if all particles carry equal charge while the vacancy is neutral, vacancy moves along a selected direction will be suppressed. In this case, the boundary conditions determine the nature of the resulting steady state. For reflecting boundary conditions, it is described by a simple Boltzmann factor, incorporating a gravitational or an electrostatic potential. Here, a new scaling variable, $\Delta \equiv L E$, emerges which characterizes the potential difference between opposing boundaries. In contrast, in the periodic case the steady state is a nonequilibrium one. Moreover, it is translation-invariant, so that all dependence on $E$ disappears in a suitably chosen co-moving frame! Remarkably, however, these boundary-induced differences appear only in the scaling functions, leaving us with a universal set of scaling 
exponents $\left\{z_{e}, z_{l}, \alpha, \sigma ; \beta\right\}[31]$.

Finally, we should add a few remarks on the early regime. As pointed out above, for an infinite system with a single vacancy, this is the only regime. First, notice that only the phenomenon of interfacial destruction is relevant, since bulk disordering would take infinitely long times. Next, considering the effective range of a Brownian walker, it is clear that the size of the disordered region grows at most with $t^{1 / 2}$. In more detail, we see that there are two distinct types of disorder. Loosely, we will label them "transverse" and "longitudinal." The first type is associated with a monolayer-like disorder from the initially sharp interface, spreading from where the vacancy began its journey. In particular, each time the vacancy crosses the plane at a new location, a particle is moved into the "wrong" phase. Thus, we may expect transverse disorder to spread like $t^{1 / 2}$. The second type is a measure of the width of the interfacial profile. For this to occur, the vacancy must "carry" e.g., a black particle deeper and deeper into the sea of white ones. Thus, the return probability of the walker plays a crucial role. Only in $d=2$ is this probability both relevant and non-trivial. Applying the well-known results of random walks [22], we find that "longitudinal" disorder grows as $(\ln t)^{1 / 2}[17]$.

Even though our model is very simple, it forms the basis for the description of a large variety of related systems. Moreover, it is truly gratifying that considerable analytic progress is possible for a problem that is both nonlinear and time-dependent. Work is in progress to probe the effect of fluctuations on the structural decomposition process at a more microscopic level [32].

\section{Acknowledgments}

The authors gratefully acknowledge fruitful collaborations with T. Aspelmeier, G. Korniss, and Z. Toroczkai. We also thank J. Beauvais, M. Grant, T. Newman, S. Redner and B. Taggart for helpful discussions. This work was supported in part by the National Science Foundation through the Division of Materials Research.

\section{Appendix: Poisson resummation}

In this Appendix, we add a few details of the Poisson resummation procedure. Our starting point is Equation (10). Dropping the constant prefactor, we focus on the expression in the brackets, with $\zeta \equiv \epsilon t$ :

$$
F(\zeta) \equiv 1-\frac{8}{\pi^{2}} \sum_{n=1}^{\infty} e^{-\zeta(2 n-1)^{2}} /(2 n-1)^{2}
$$

This sum converges rapidly in the limit $\zeta \rightarrow \infty$. We seek a form which converges equally well in the limit $\zeta \rightarrow 0$. We first recast this sum in terms of a much simpler one, namely,

$$
S(\lambda) \equiv \sum_{n=1}^{\infty} e^{-(\lambda n)^{2}}
$$

We begin by noting that $\sum_{1}^{\infty} 1 /(2 n-1)^{2}=\pi^{2} / 8$ so that

$$
\begin{aligned}
F(\zeta) & =\frac{8}{\pi^{2}} \sum_{n=1}^{\infty} \frac{1-e^{-\zeta(2 n-1)^{2}}}{(2 n-1)^{2}} \\
& =\frac{8}{\pi^{2}} \zeta \int_{0}^{1} d s \sum_{n=1}^{\infty} e^{-\zeta(2 n-1)^{2} s}
\end{aligned}
$$

It is easy to recognize the sum in this expression as the "odd" terms in $S(\lambda)$, with $\lambda=\sqrt{s \zeta}$. This suggests that we should recast $S(\lambda)$ in the form

$$
\begin{aligned}
S(\lambda) & =\sum_{n=1}^{\infty} e^{-(2 \lambda n)^{2}}+\sum_{n=1}^{\infty} e^{-(\lambda(2 n-1))^{2}} \\
& \equiv S_{e}(\lambda)+S_{o}(\lambda)
\end{aligned}
$$

where the "even" terms just reproduce $S(\lambda)$, via $S_{e}(\lambda)=S(2 \lambda)$. Summarizing so far, we obtain

$$
\begin{aligned}
F(\zeta) & \equiv \frac{8}{\pi^{2}} \zeta \int_{0}^{1} d s S_{o}(\sqrt{\zeta s}) \\
& =\frac{8}{\pi^{2}} \zeta \int_{0}^{1} d s[S(\sqrt{\zeta s})-S(2 \sqrt{\zeta s})]
\end{aligned}
$$

Thus, it is sufficient for us to resum $S(\lambda)$. We recall the Poisson resummation formula [27], for a function $f(x)$, defined on $0 \leq x<\infty$ with $\lim _{x \rightarrow \infty} f(x)=0$ and $\int_{0}^{\infty} d x f(x)$ finite:

$$
\frac{1}{2} f(0)+\sum_{n=1}^{\infty} f(n \lambda)=\lambda^{-1}\left[\frac{1}{2} \tilde{f}(0)+\sum_{m=1}^{\infty} \tilde{f}\left(\frac{2 \pi m}{\lambda}\right)\right]
$$

where $\tilde{f}$ is the Fourier cosine transform of $f$ :

$$
\tilde{f}(\alpha) \equiv 2 \int_{0}^{\infty} d x f(x) \cos (\alpha x) .
$$

From Eqns (14), we can immediately read off $f(x)=\exp \left(-x^{2}\right)$ for our case whence

$$
\tilde{f}(\alpha)=\sqrt{\pi} \exp \left(-\alpha^{2} / 4\right) \text {. }
$$


Thus, we obtain

$$
S(\lambda)=-\frac{1}{2}+\lambda^{-1}\left\{\frac{\sqrt{\pi}}{2}+\sqrt{\pi} \sum_{m=1}^{\infty} e^{-(m \pi / \lambda)^{2}}\right\}
$$

Clearly, the original form of $S(\lambda)$, Eq. (14) converges rapidly for large $\lambda$, while the alternate form presented here converges rapidly for $\lambda \rightarrow 0$. Inserting our result into Eq. (16) and performing the integration results in

$$
F(\zeta)=\frac{4}{\pi^{3 / 2}} \sqrt{\zeta}\left\{1+2 \sum_{m=1}^{\infty}(-1)^{m}\left[e^{-u_{m}^{2}}-u_{m} \Gamma\left(\frac{1}{2}, u_{m}\right)\right]\right\}
$$

where $u_{m} \equiv \pi m / \sqrt{8 \epsilon t}$. This leads immediately to our result for the disorder parameter, Eq. (11).

The Poisson resummation of the magnetization profile, Eq.(8), is a little more involved, but follows an analogous series of steps. In this case, the problem can be reduced to resumming

$$
\Sigma(\tilde{y}, \zeta) \equiv \sum_{n=1}^{\infty} \frac{\sin (n \tilde{y})}{n} e^{-\zeta n^{2}}
$$

where $\tilde{y} \equiv 2 \pi y / L$, since we can rewrite

$$
\psi(\mathbf{r}, t)=\frac{4}{\pi}\left[\Sigma(\tilde{y}, \zeta)-\frac{1}{2} \Sigma(2 \tilde{y}, 4 \zeta)\right] .
$$

The resummation leads to

$$
\Sigma(\tilde{y}, \zeta)=-\frac{\tilde{y}}{2}+\frac{\pi}{2} \operatorname{erf}\left(\frac{\tilde{y}}{2 \sqrt{\zeta}}\right)+\frac{\pi}{2} \sum_{m=1}^{\infty}\left[\operatorname{erf}\left(\frac{\tilde{y}+2 \pi m}{2 \sqrt{\zeta}}\right)+\operatorname{erf}\left(\frac{\tilde{y}-2 \pi m}{2 \sqrt{\zeta}}\right)\right]
$$

so that we finally arrive at Eq. (12).

\section{References}

[1] J.D. Gunton, M. San Miguel and P.S. Sahni, in: Phase Transitions and Critical Phenomena, Vol. 8, edited by. C. Domb and J.L. Lebowitz (Academic, New York, 1983); H. Furukawa, Adv. Phys. 34, 703 (1985); K. Binder, Rep. Prog. Phys. 50, 783 (1987), and in: Phase Transitions of Materials, edited by R.W. Cahn, P. Haasen and E.J. Kramer (VCH, Weinheim, 1991); J. S. Langer, in Solids far from Equilibrium\}, edited by C. Godrèche (Cambridge University Press, 1992); A. J. Bray, Adv. Phys. 43, 357 (1994).

[2] See, e.g., W.D. Callister Jr., Material Science and Engineering: An Introduction (Wiley, 1994).

[3] M. Blume, V.J. Emery and R.B. Griffiths, Phys. Rev. A 4, 1071 (1971); R.B. Potts, Proc. Camb. Phil. Soc. 48, 106 (1952); F.Y. Wu, Rev. Mod. Phys. 54, 235 (1982).

[4] K. Yaldram and K. Binder, Acta metall. mater. 39, 707 (1991), J. Stat. Phys. 62, 161 (1991) and Z. Phys. B82, 405 (1991); K. Yaldram, G.K. Khalil and A. Sadiq, Solid State Comm. 87, 1045 (1993); P. Fratzl and O. Penrose, Phys. Rev. B50, 3477 (1994) and Phys. Rev. B 55, R6101 (1997).

[5] S. Puri, Phys. Rev. E 55, 1752 (1997); S. Puri and R. Sharma, Phys. Rev. E 57, 1873 (1998).

[6] K.W. Kehr, K. Binder, and S.M. Reulein, Phys. Rev. B 39, 4891 (1989).
[7] S. Alexander, Y. Rabin, and R. Zeitak, J. Chem. Phys. 95, 2012 (1991).

[8] O.G. Mouritsen and P.J. Shah, Phys. Rev. B 40, 11445 (1989); P.J. Shah and O.G. Mouritsen, Phys. Rev. B 41, 7003 (1990); E. Vives and A. Planes, Phys. Rev. Lett. 68, 812 (1992) and Phys. Rev. B 47, 2557 (1993); C. Frontera, E. Vives, and A. Planes, Phys. Rev. B 48 , 9321 (1993); C. Frontera, E. Vives, T. Castan and A. Planes, Phys. Rev. B 51, 11369 (1995) and Phys. Rev. B 55, 212 (1997); M. Porta, C. Frontera, E. Vives, and T. Castan, Phys. Rev. B 56, 5261 (1997); C. Frontera and E. Vives, Phys. Rev. B 59, 11121 (1999); M. Porta, E. Vives, and T. Castan, Phys. Rev. B 60, 3920 (1999).

[9] M. Plapp and J.-F. Gouyet, Phys. Rev. Lett. 78, 4970 (1997).

[10] I. Žižak, P. Fratzl, and O. Penrose, Phys. Rev. B 55, 12121 (1997).

[11] H-F. Meng and E.D.G. Cohen, Phys. Rev. E 51, 3417 (1995); J. Krug and P. Meakin, Phys. Rev. Lett. 66, 703 (1991); R. Cuerno, H.A. Makse, S. Tomassone, S. Harrington and H.E. Stanley, Phys. Rev. Lett. 75, 4464 (1995) and references therein; S. G. Corcoran, in Proceedings of the Electrochemical Society, Symposium on Critical Factors in Localized Corrosion III, 500 (1999).

[12] D. Drouin, J. Beauvais, R. Lemire, E. Lavallée, R. Gauvin, and M. Caron, Appl. Phys. Lett. 70, 3020 (1997). 
[13] M. N. Baibich, J. M. Broto, A. Fert, F. Nguyen Van Dau, F. Petroff, P. Etienne, G. Creuzet, A. Friederich, and J. Chazelas, Phys. Rev. Lett. 61, 2472 (1988); G. Binasch, P. Grünberg, F. Saurenbach, and W. Zinn, Phys. Rev. B 39, 4828 (1989).

[14] S. Sanvito, C.J. Lambert, and J.H. Jefferson, condmat/9903381 (1999).

[15] Y. Ohno, Y. Kawai and S. Takeda, Phys. Rev. B , 59, 2694 (1999).

[16] M. J. A. M. Brummelhuis and H. J. Hilhorst, J. Stat. Phys. 53, 249 (1988). See also Z. Toroczkai, Int. J. Mod. Phys. B 11, 3343 (1997).

[17] R.K.P. Zia and Z. Toroczkai, J. Phys. A 31, 9667 (1998).

[18] Z. Toroczkai, G. Korniss, B. Schmittmann and R. K. P. Zia, Europhys. Lett. 40, 281 (1997).

[19] L. Onsager, Phys. Rev. 65, 117 (1944).

[20] N. Metropolis, A.W. Rosenbluth, M.M. Rosenbluth, A.H. Teller, and E. Teller, J. Chem. Phys. 21, 1087 (1953).

[21] B.M. McCoy and T.T. Wu, The Two-Dimensional Ising Model (Harvard University Press, 1973).

[22] B. D. Hughes, Random Walks and Random Environments, Vol. 1: Random Walks (Clarendon Press, Oxford, 1995).
[23] S.T. Chui and J.D. Weeks, Phys. Rev. Lett. 40, 733 (1978); S.F. Edwards and D. R. Wilkinson, Proc. Roy. Soc. London, Ser. A 381, 17 (1982).

[24] M. Kardar, G. Parisi, and Y.-C. Zhang, Phys. Rev. Lett. 56, 889 (1986).

[25] B. Schmittmann, K. Hwang, and R.K.P. Zia, Europhys. Lett. 19, 19 (1992).

[26] D.J. Amit, Field Theory, The Renormalization Group and Critical Phenomena (World Scientific, 1984).

[27] D.S. Jones, The Theory of Generalised Functions (Cambridge University Press, 1982).

[28] H. van Beijeren and I. Nolden, in Structure and Dynamics of Surfaces II, edited by W. Schommers and P. von Blanckenhagen; Vol. 43 of Topics in Current Physics (Springer Verlag, Berlin, 1987).

[29] J.S. Rowlinson and B. Widom, Molecular Theory of Capillarity (Oxford University Press, 1982).

[30] B. Schmittmann, unpublished.

[31] W. Triampo, T. Aspelmeier, and B. Schmittmann, cond-mat/9910275 (1999).

[32] T. Aspelmeier, B. Schmittmann, W. Triampo and R.K.P. Zia, to be published. 